Enas G Younis

BDS, MSc ( Asst. Lec.)

\section{Study the Temperature Effects on Mechanical Properties of Cold Cured Denture Base Acrylic Material}

Dept of Prosthetic Dentistry

College of Dentistry, University of Mosul

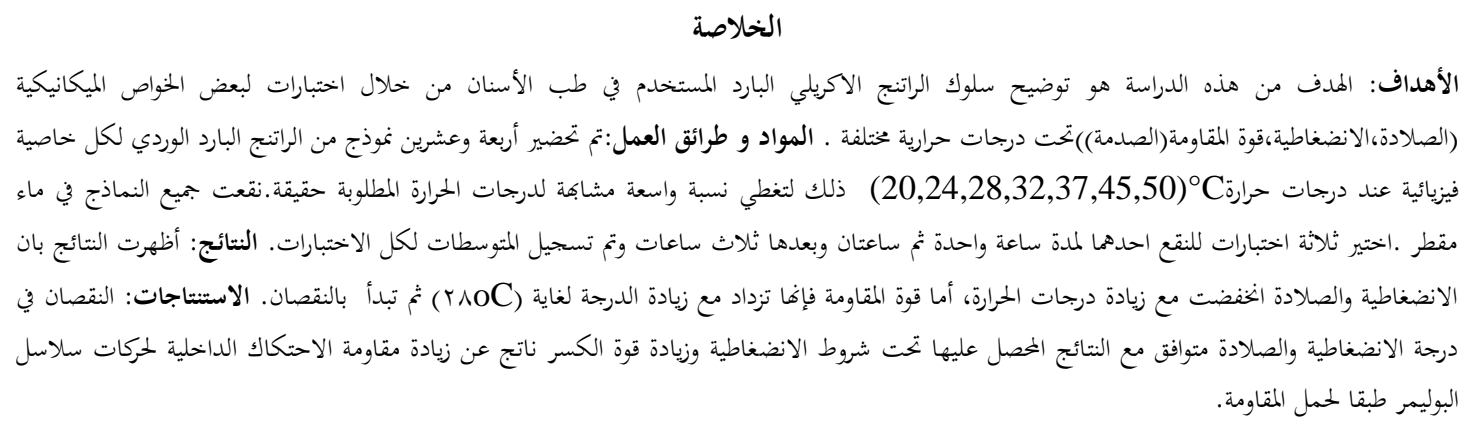

ABSTRACT

Aims: The purpose of this study is to explore the behavior of cold curing acrylic resin which used in dentistry during some mechanical properties (hardness, compression and impact)tests under different temperatures. Materials and Methods: Twenty four pinks from cold cured samples for each mechanical-physical properties tested were prepared under temperatures $(20,24,28,32,37,45,50) \mathrm{C} 0$ to cover a wide range similar to actual service temperatures. All specimens were soaked in distilled water. One hour ,two hours ,three hours were chosen as the time for soaking and the means of the tests were recorded. Results: The results show that the compression and hardness decreasing with the increasing of temperature and increase of impact strength with the increase of temperature up to (28) C0 then followed by a decrease in impact strength. Conclusions: The decrease of compressive strength and hardness is compatible with the results obtained under compressive Conditions.

Keywords: Temperature, mechanical properties, cold cured material.

Younis EG. Study the Temperature Effects on Mechanical Properties of Cold Cured Denture Base Acrylic Material. Al-Rafidain Dent J. 2013; 13(2): 346-350.

Received: $2 / 5 / 2012$

Sent to Referees: $7 / 5 / 2012$

Accepted for Publication: 18/6/2012

\section{INTRODUCTION}

The suitability of any material for structural applications may be determined by mechanical tests. Such testing includes a determination of the ability of a material to with stand various stress systems which 'may be tensile, compressive, shear or cyclic in nature. The behavior of any material is also influenced by the presence of defects, the intrinsic micro structural features and the rate of application of stress, which may vary from exceedingly slow rates of deformation to impact loading. ${ }^{(1,2)}$

Cold-cured acrylic resin is one of the most frequently used materials in dentistry for repairs, relines, orthodontic appliances, maxillofacial prosthesis in addition to its use in crown and bridge work as a temporary coverage of prepared tooth..$^{(3-11)}$

An optimized resin material should exhibit a positive biologic response while maintaining the desired physical properties. Physical and mechanical properties of polymers are crucial in achieving clinical success and longevity of complete dentures fabricated. Important physical properties include the following: compressive and tensile strengths, elongation, hardness, thermal characteristics, molding properties, polymerization shrinkage, solubility, dimensional stability, and dimensional accuracy. ${ }^{(4-13)}$

Denture base fractures have been examined using different testing protocols. Strength testing can include compressive, shear, tensile, transverse, impact, and fatigue strength. Earlier study exhibited some physical properties that warrant further investigation. Micro cracks were observed under light microscope, especially in modified PMMA samples that had higher meth acrylic acid content suggest- 
ing that increasing the ratio of meth acrylic acid may compromise the physical properties of the resin. ${ }^{(9,10)}$ In light of these findings, it is important to elucidate the physical properties of these surface-modified resins. ${ }^{(5-12)}$

Because heat affects maturity of the chemical reaction of acrylic resin, therefore, its maintenance during polymerization may affect the mechanical properties of cured resin. ${ }^{(6-14)}$

At the higher temperatures the specimen deforms plastically prior to catastrophic failure. At the lower temperatures, the specimen exhibits little or no plastic deformation and fails predominantly by brittle ineer-crystalline cleavage. In the transition zone between these two extremes, the material fails by a mixture of ductile and brittle fracture. A transition temperature, $\mathrm{T}^{\mathrm{o}} \mathrm{c}$, sometimes taken as the temperature in the. middle of the transition zone characterizes this behavior of a material for a given testing condition. To protect a component from brittle failure it is necessary that the transition temperature of the material be below the lowest expected service temperature impact testing. Since this represents a high strain rate and since the transition temperature is raised by increasing the strain rate, Using this characteristic of the material helps to ensure that the structure will not undergo brittle failure. . $^{(1,7,8,13)}$

This paper is devoted to explore the behavior of a widely used cold cured acrylic denture base material during mechanical properties(hardness, compression and impact) tests at different temperatures to determine the relative resistance of acrylic resin.

\section{MATERIALS AND METHODS}

A- Specimens grouping:

Seventy-two specimens were prepared from pink cold-cure acrylic resin denture base .The study includes three groups of specimens depending on the method of processing of the cold-cure acrylic resin and each group of them contain 24 specimens for each test. Pink cold cure acrylic resin(RESPAL-NF liquid - Pro Base cold powder) was mixed according to manufacturer's instruction $(1: 2)$ by volume. ${ }^{(9,10)}$

\section{$B$-Impact Tests}

This test aims at subjecting the denture to sudden load. Charpy's pendulum impact testing machine(ZEIZZ-West Germany) (Fig1) was used to determine the relative impact resistance of acrylic resin at different temperatures. The machine is a pendulum-type with a disc-shaped hammer carrying a knife edge. Rectangular specimens were prepared ( $40 \mathrm{~mm}$ long, $5 \mathrm{~mm}$ in both thickness and width), each having a $1 \mathrm{~mm}$ deep standard notch with $60^{\circ}$ angle in the middle of the bar. All specimens were immersed in distilled water before tested. The specimen was simply supported horizontally at both ends and not clamped. The impact strength means of a specimen was recorded at temperatures $(20,24,28,32$, $37,45,50) \mathrm{C}^{0}$ as the number of $\mathrm{kg} . \mathrm{mm}$ of energy absorbed in breaking the specimen. The specimens must be changed at each temperature degree after each test.

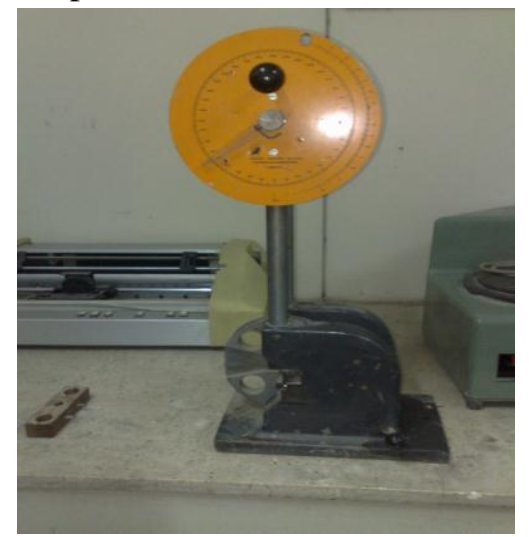

Figure (1): Charpy's pendulum impact testing machine (ZEIZZ-West Germany)

C-Compressive Strength Test:

Specimens for this test were in the form of a cylindrical with dimen- sions $(20 \mathrm{~mm} \times 10 \mathrm{~mm})$ were subjected to compressive load until failure, using a universal testing machine (Figure 2). 


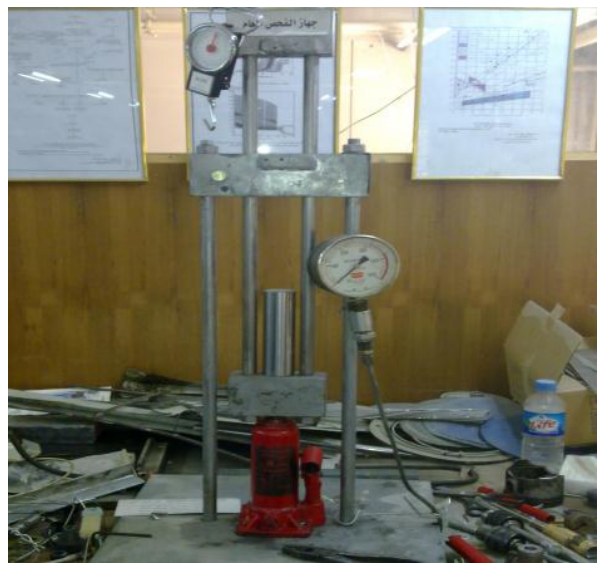

Figure (2): universal testing machine for compressive strength test

All specimens were immersed in distilled water before tested. Each specimen was placed between two parallel flat surfaces and the compressive load was gradually increased until sample failure occurred. Great care was required to insure proper transfer of load from testing machine to specimen. The load was made axially and applied uniformly over the end of the specimen. The compressive strength means calculated at temperatures $(20,24$, $28,32,37,45,50) \mathrm{C} 0$ as stress in $\mathrm{N} / \mathrm{mm} 2$ from the following equation:

Compressive Strength $=$ F/A

$\mathrm{F}$ : Force at failure (Newton)

A : Minimum cross sectional area ( $\mathrm{mm} 2)$ (The specimens must be changed at each temperature degree after each test).

\section{D- Hardness test}

Specimens were prepared in the form of a spherical disk with diameter $(14 \mathrm{~cm})$ ,(Amsler,KARL-KOLB/Germany) Rockwell tester was used in this study for measuring the indentation hardness(fig 3).

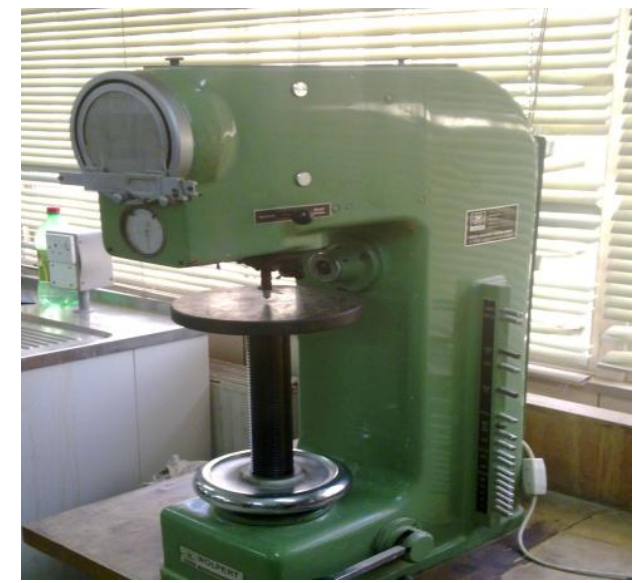

Figure (3): (Amsler,KARL-KOLB/Germany) machine for measuring the indentation hardness.

All specimens were immersed in distilled water before tested. The test load was set from $(10$ to $60 \mathrm{Kg})$. The contact period between the specimen and the indenter was six seconds. After that the measurement were taken directly from the scale, three reading were done on different areas of each specimen and the mean of three reading was calculated. Hardness calculated at temperatures $(20,24,28,32$, $37,45,50)$ C0.The specimens must be changed at each temperature degree after each test.

\section{RESULTS AND DISCUSSION}

Impact strength, hardness and compressive strength results are shown in Table (1). 
Table (1): The (impact strength, hardness and compressive strength)means under different temperatures.

\begin{tabular}{cccc}
\hline TemperatureT $\left(\mathrm{c}^{0}\right)$ & Hardness Test HRR & Compression Test N/mm & Impact Test Kg.m \\
\hline 20 & 119 & 345 & 75.5 \\
24 & 118 & 337 & 73 \\
28 & 117 & 332 & 71.5 \\
32 & 116 & 322 & 69.5 \\
37 & 115 & 312 & 69 \\
40 & 115 & 305 & 68.5 \\
45 & 112 & 293 & 65.2 \\
50 & 111 & 260 & 64.6 \\
\hline
\end{tabular}

Statistical analysis of data by using analysis of variance "ANOVA" test revealed that there is a statistically signifi- cant difference $(p<0.001)$ between the temperature among the three groups, as shown in Table (2).

Table (2): Analysis of Variance (ANOVA) test for(impact strength, hardness and compressive strength) groups.

\begin{tabular}{llllll}
\hline $\begin{array}{l}\text { Source of Var- } \\
\text { iance SOV }\end{array}$ & $\begin{array}{l}\text { Degree of } \\
\text { freedom DF }\end{array}$ & $\begin{array}{l}\text { Sum of } \\
\text { Squares SS }\end{array}$ & $\begin{array}{l}\text { Mean of } \\
\text { squares MS }\end{array}$ & F-value & $p$-value \\
\hline Factor & 3 & 388025 & 129342 & $1186.80 *$ & 0.000 \\
Error & 28 & 3052 & 109 & & \\
Total & 31 & 391076 & & & \\
\hline
\end{tabular}

\section{Impact tests}

The variation of Charpy impact energy with temperature is given in (Figure 4). The results did not show a clear transition temperature similar to metals. However, a slight increase of fracture energy was developed with the increase of temperature then followed by a decrease in fracture energy with further increase of tempera- ture. An explanation of fracture energy increase is due to the increase of internal frictional resistance of polymer chains movement due to the impact load. This movement becomes easier at higher temperatures. which contribute to a decrease in fracture energy with further increase in temperature.

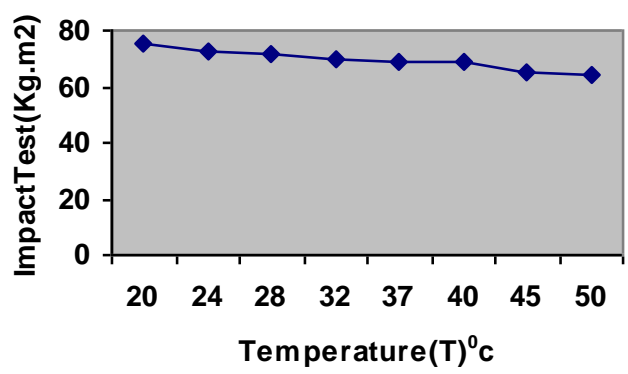

Figure (4):The variation of Charpy impact energy with temperature.

\section{Hardness and Compressive Strength tests}

The variation of hardness and compressive strength values of the cold-cure acrylic resin samples with temperature is given in Figures $(5,6)$. As expected, the material gives higher hardness values at lower temperature. The difference in the actual value of hardness and compressive 
strength in indentation and scratch for each temperature is due to the difference in strain rate applied during both tests. An explanation of fracture energy increase is due to the increase of internal frictional resistance of polymer chains movement due to the impact load. This movement becomes easier at higher temperatures, which contribute to a decrease in fracture energy with further increase in temperatures.

\section{CONCLUSIONS}

The Conclusions of this study shows a decrease of the compressive strength and hardness as the temperature is increasing. In the same range of temperature. The decrease of compressive strength and hardness is compatible with the results obtained under compressive conditions and a slight increase of fracture energy with increase of temperature then followed by a decrease in fracture energy with further increase of temperature.

\section{REFERENCES}

1. Al-Neami ZJ. "The effect of different water temperatures during polymerization on some physical and mechanical properties of cold-cure acrylic resin materials". M Tech Thesis, Dental Technologies in the College of Health and Medical Technology. (2005)

2. Al-Musawi RM."Evaluation of Glycerin as a Separating Medium For Processing Acrylic Denture Base Materials" ((Comparative Study)) Msc Thesis, Dental Technologies in the College of Health and Medical Technology. (2005)

3. S. E. Park, AR Periathamby, and JC Loza,(2003) "Effect of surfacecharged poly (methyl methacrylate) on the adhesion of Candida albicans," $J$ Prosthodontics, vol. 12, no. 4, pp. 249-25.

4. Kamil NB.(2008)"Effect of Addition of Different Length and Concentration of Silane Treated Glass Fibers on Some Properties of Heat cured Acrylic Resin "M sc Thesis, Department of Prosthodontics, University of Baghdad..
5. Raghdaa K. Jassim. B. Aseel A. Radhi. B. (2011):" Evaluation the biological effect of two types of denture base materials reinforced with silanated glass fiber" J Bagh College Dentistry Vol. 23(2),

6. Măries G.R, Bandur G. Rusu G (2008):" Study by Thermal Methods of Some Physical-Mechanical Properties of Polypropylene (PP) Used for High Performance Sport Products " Chem. Bull. "POLITEHNICA" Univ. (Timisoara) Volume 53(67), 1-2,

7. Tillová, E., Duriníková, E., Chalupová, M. (2011):" Materials Engineering"18, pp. 1-7 ISSN 1335-0803.

8.Sahin S., Yayla P.,(2005):"Effects of testing parameters on themechanical properties of polypropylene random copolymer"Polym. Test 24 613-619.

9. Bernardi MIB, Rojas SS, Andreeta MRB, Rastelli ANS, Hernandes AC, BagnatoVS.(2008):"Thermal analysis and structural investigation of different dental composites resins" $J$ Therm Anal Calorim.;94:791-6.

10. Conti C, (2005):" Spectroscopic and mechanical properties of dental resin composites cured with different light sources" J Mol Struc;744-747:641-6.

11. Kele_temur O, Yildiz S. (2009):" Effect of various dual-phase heat treatments on the corrosion behavior of reinforcing steel used in the reinforced concrete structures" Const. Build. Mat., 23(1): 78-84.

12. Yilmazer B. (2009):" Researching physical and mechanical properties of heavy concrete produced using barite" $M$ sc Thesis, University of Firat, (in Turkish).

13. Akkurt I, Altindag R, Basyigit C, Kilincarslan S. (2008):"The effect ofbarite rate on the physical and mechanical properties of concretes under F-T cycle" Mater Desg. 29(9): 1793-1795.

14. Kele_temur O, Aksoy M,Yildiz S. (2009):"Corrosion behavior of tempered dual-phase steel embedded in concrete" Int. J Min Met Mat. 16(1): 43-50 